\title{
Evaluating Contact Detection, Size Recognization and Grasping State of an Object using Soft Elastomer Gripper
}

\author{
Kazi Abaul Jamil \\ Department of Automation \\ School of Electronics, Information and Electrical Engineering \\ Shanghai Jiao Tong University
}

\begin{abstract}
Object handling process of the sensitive or fragile object is critical to preserve its quality. In this domain, soft robotics has gained a lot of attention. However, limitations of detecting contact and grasping behavior is still a challenging task due to the non-linear behavior of soft gripper. Moreover, to regulate grasping behavior, exact real-time contact feedback is a crucial task. To improve the contact detection accuracy a gradient-based algorithm is proposed with the feedback from a simple resistive flex sensor and a pressure sensor. For that purpose firstly, the resistive flex sensor is embedded into the gripper to get the real-time gripper's finger position. Secondly, solenoid valves and pressure sensors are used to control the pneumatic pressure from the pump, and finally, a closed-loop control system is developed for controlling the grasping process. The proposed contact detection algorithm can provide contact feedback with an accuracy of $\pm 3 \mathrm{~mm}$, which is implemented to perform the size recognization of the sphere-shaped objects. A real-time experimental setup has been developed which can successfully perform a pick and place of fruits and vegetables. The key benefits of the proposed algorithm are less complexity and better accuracy.
\end{abstract} tion

Keywords-Grasping process; contact feedback; size recogniza-

\section{INTRODUCTION}

In the recent few years, soft robotics has gained a lot of consideration due to its unrivaled agility, compliance and safe interactions with objects [1] [2]. Robotic manipulation without degrading the quality of sensitive, fragile or deformable objects quality is a challenging task. The soft gripper has wide applications in automated industries such as safe human-robot interaction [3], minimally invasive surgery [4], harvesting and food handling [5], [6]. Various soft actuators are introduced using pneumatic, cables, fluid, motor or magnetic fields. Embedding additional sensors to this type of actuator is challenging and makes the system bulky and incompact. Accurate observation of contact detection and grasping behavior by image processing is perplexing task [7], [8]. Moreover, grasp target information i.e size or position remains unidentified due to opaqueness or obstacles between the gripper. Such grippers are difficult to regulate the excessive contact force for sensitive objects [6]. The contact detection is very important for sensitivity analysis during grasping. The interface of contact detection with an object may perform different grasping related operations i.e. object geometry recognition, size recognition and pose estimation. A deep convolutional network algorithm is proposed where a soft robot grasps the object using camera [9]. In [6], [7], [10] the concept of contact detection is discussed, whereas real implimentation is still challenging. A clustering method is implemented to identify a set of irregular shape objects based on the internal state of the finger [11]. This method is limited to contact detection or size recognition of the grasped object. In order to experience the real-time proprioceptive grasping, flexible and stretchable sensors embedded with soft bodies are fabricated and designed [12] [13]. Implemented pressure and curvature sensing of soft actuators in a closed loop system represents the concept of soft haptic perception based on the piezoresistive effect [14].

The characteristics of linearity, robustness, compactness, low power consumption, the flexibility of commercially available resistive flex sensors suits to integrated with the soft actuators [15]. Whenever soft acutuator bends the resisitivity of flex sensor changes. This technique is utilized to estimate the internal state of the fingers of the gripper and perform the haptic identification of grasped objects [16]. The data-driven approach was implemented to estimate the bending angle of the soft actuator based on flex sensor value and onboard pressure sensor value [17]. However, this method needs to collect a lot of experimental data for calibration of each actuator. Thus, to overcome this problem, in [6] a linear response of object size and flex sensor reading are analysed from realtime raw feedback sensor data for evaluating contact and size of objects. In [18] contact detection and size recognition of round shape objects are performed comparing bend sensor and pressure sensor data. The bending state has been measured by comparing current bend value with free bend value which is difficult to calibrate when source pressure value is varying.

To the best of the author's knowledge, there is a need for an alternative approach that can overcome the limitations of realtime monitoring of the bending state. The prime contributions of this study are listed below:

- A contact detection algorithm has been proposed based on the gradient response of a commercially available embedded resistive flex sensor. The contact detection algorithm is validated using the touch sensor in the experiments.

- A size recognition algorithm has been proposed considering sphere-shape objects based on raw sensory contact feedback.

- The grasping state of the object is analysed using 
raw sensory feedback ensuring whether the object is grasped or released, which helps to reduce the grasp uncertainty or delay in soft robust grasping.

- Proposed algorithm is capable to monitor the real-time bending state of the actuator during grasping of the target object.

- Key benefits of the proposed setup and algorithm are less complexity, better accuracy, and lower cost.

The rest of the paper is organized as follows: Section II describes the experimental design including the fabrication of soft gripper, hardware setup, and noise filter design. The methods of the proposed algorithm are discussed in Section III. Section IV discusses the experiments performed using the proposed method. The results of the performed experiment are discussed in Section V. In Section VI, an automated fruit sorting environment is implemented to perform a realtime sorting process. Section VII concludes how the proposed methods are used to preserve the quality of sensitive or fragile objects.

\section{EXPERIMENTAL DESIGN}

\section{A. Design of Soft Robotic Gripper}

The design of the gripper is considered from open source design (https://softroboticstoolkit.com) with customized soft gripper, a size of $150 * 20 * 10 \mathrm{~mm}$ is prepared by using smooth on Smooth-On Ecoflex-30 material as shown in Fig. 1. Additionally, a flex sensor of length $11.5 \mathrm{~cm}$ is embedded into the base of the soft gripper as shown in Fig. 2 to perform the contact detection and size recognition. During validation of the proposed contact detection algorithm, a touch sensor is embedded at the fingertip of the soft gripper, whose measurement is considered as a ground truth.

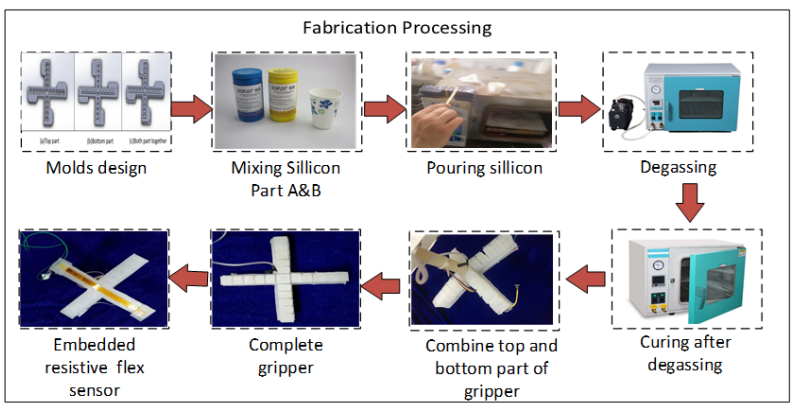

Fig. 1. Fabrication Process

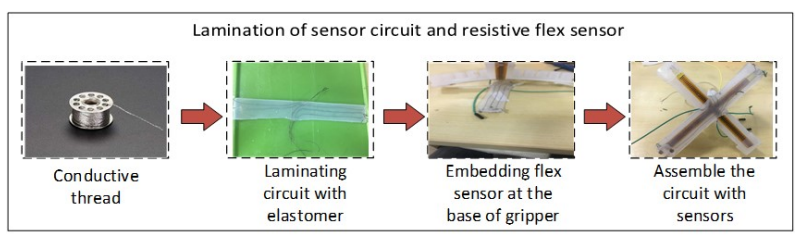

Fig. 2. Embedding Resistive Flex Sensor

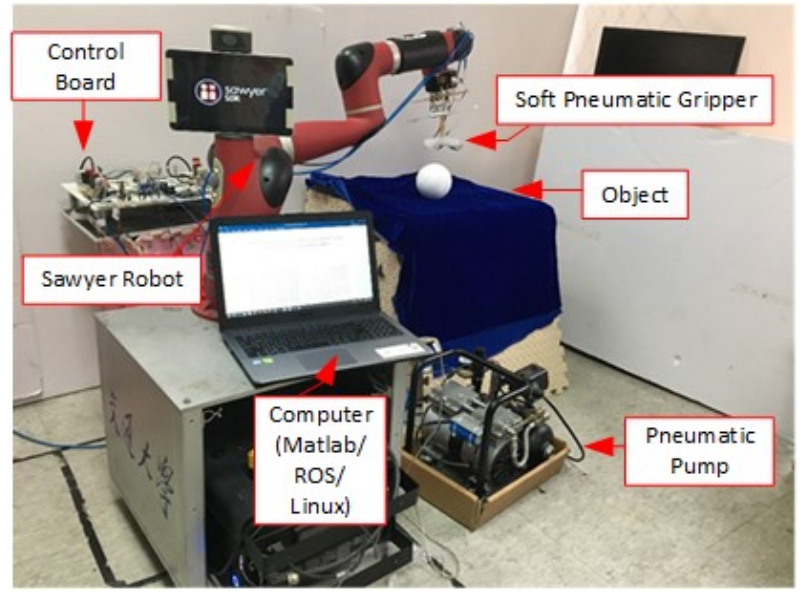

Fig. 3. Diagram of the Experiment Setup

\section{B. Hardware Setup}

An experimental setup has been constructed systematically to perform the grasping test as shown in Fig. 3.

- $\quad$ Soft gripper: Gripper with embedded flex sensor.

- Sawyer robot: To perform pick and place of an object.

- 3D printed support: A 3D printed support is mounted at the end-effector of sawyer robot to facilitate the pneumatic soft gripper.

- Control board: A closed loop control is implemented to obtain the required pressure for grasping operation. It consists of a microcontroller board(Arduino Uno), solenoid valves (Delixi solenoid valve, 4v410 DC 24V), MOSFET switch module (SZH-AT021, SZH), and pressure sensor(100PGAA5, Honeywell).

- Pneumatic supply: Pneumatic pump with 0.82 gal cylinder is used for pneumatic supply.

- Computer: Linux OS, Arduino, Matlab software, robotic operation system (ROS).

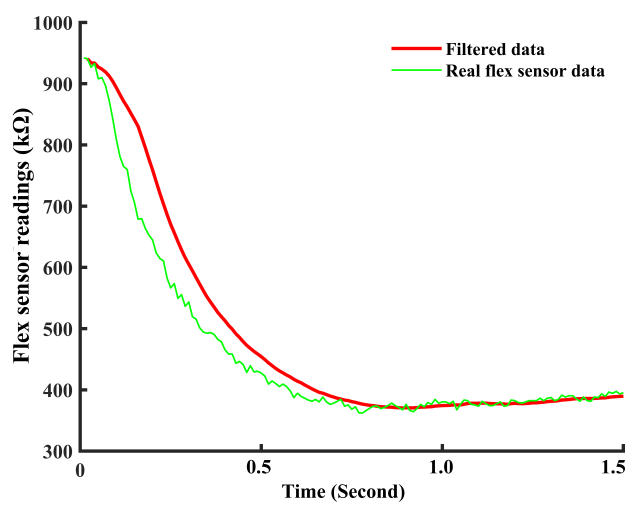

Fig. 4. Implementing Moving Average Filter Data

Implementation of small pneumatic tank as a pneumatic capacitor and a sponge inside the pneumatic channel as a resistors 
are introduced to smoothen the real-time data obtained from flex sensor [17].

As the hardware setup was not enough to obtain smooth data, a moving average filter is introduced given by equation (1).

$$
y[i]=\frac{1}{m} \sum_{j=0}^{m-1} x[i+j]
$$

Where, $m=$ window size; $x=$ sensor value array; $i=$ filtered sample point; $j=$ real sensor sample point As shown in Fig. $4, m=20$ samples are considered as a window size in each iteration.

\section{METHODS}

In this section, the flex sensor response at different source pressure are discussed. A contact detection algorithm with flex sensor is proposed and a size recognition algorithm is described to perform the sorting task of sphere-shaped objects.

\section{A. Characteristics of Flex Sensor}

As the pneumatic pressure increases the flex sensor embedded inside the finger bends and gives a continuous change in resistance.To analyse the characteristics of the flex sensor, two different types of grasping are performed, i) free grasping and ii) object grasping. In free grasping no object is kept between the fingers and the sensor reading with free grasping is shown in Fig. 5 at different inflation pressure from $137 \mathrm{kPa}$ to 193 $\mathrm{kPa}$, respectively. Keeping the source pressure constant, a free

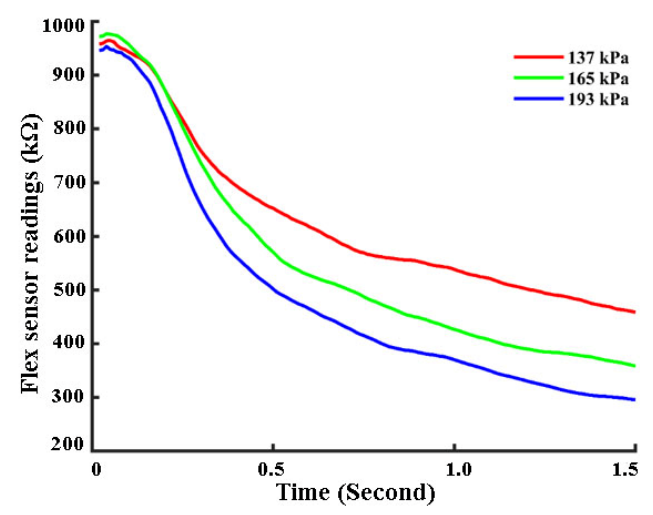

Fig. 5. Flex Sensor Readings during Free Grasping

grasping and an object grasping are performed in Fig.6 which shows the deviation of sensor response of object grasping from free grasping.

\section{B. Contact Detection}

In this study, it is observed the change in slope of flex sensor reading helps to identify the contact with the target object. For a function of $N$ variables, $F(x, y, z, \ldots)$, the gradient is defined in equation (2),

$$
\nabla F=\frac{\partial F}{\partial x} \hat{i}+\frac{\partial F}{\partial y} \hat{j}+\frac{\partial F}{\partial z} \hat{k}+\ldots \ldots \ldots+\frac{\partial F}{\partial N} \hat{n}
$$

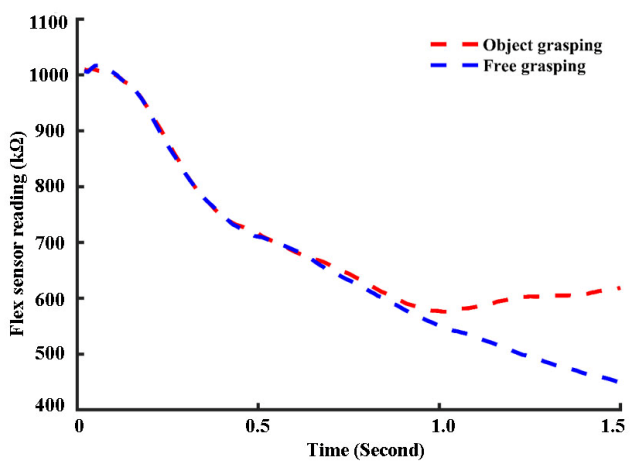

Fig. 6. Comparison of Object Grasping and Free Grasping

Based on gradient theory, contact point detection on one dimensional flex sensor data is used to derive from equation (2), where $\nabla F$ defined as the change in flex sensor value $\partial F$ to sample number $\partial x$ as in equation (3).

$$
\nabla F=\frac{\partial F}{\partial x} \hat{i}
$$

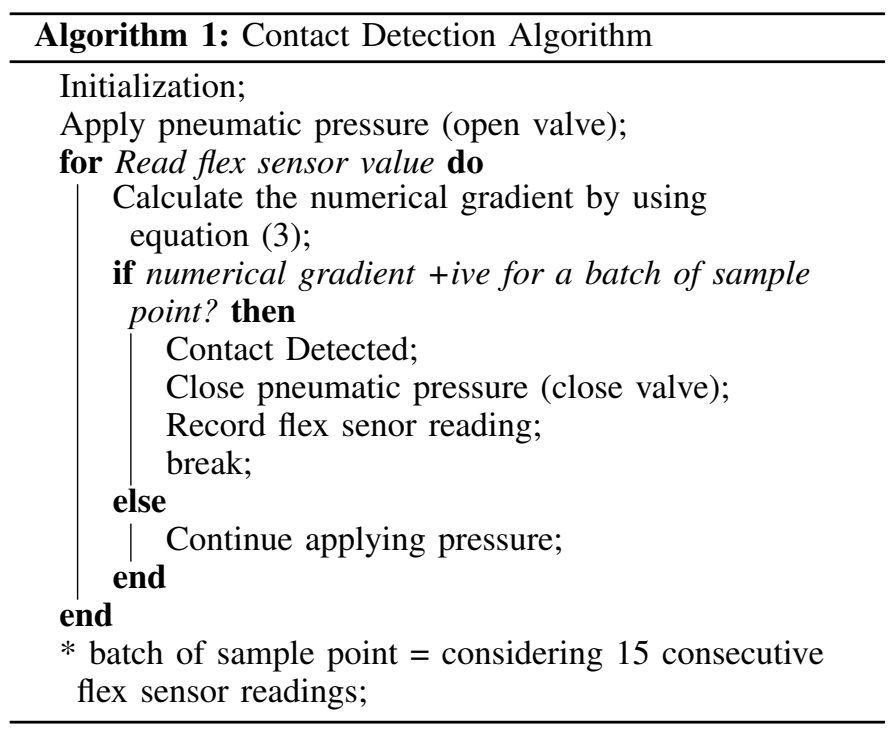

\section{Size Recognisation Algorithm}

Through contact detection algorithm it can be analyzed that different size objects results a unique value of flex sensor, which shows the capability of size recognition of object.To demonstrate the size recognition ability of SPG, objects of different sizes and materials are grasped. A human hand during grasping an object, firstly it forms enveloping grasping and analyze the shape and dimension of object and then power grasps to pick the object.When the tip of the SPG touches the object the whole gripper is ready to grasp in envelope type. A suitable pneumatic pressure is provided within working pressure range to confirm grasping stability, and grasping state are maintaining for a period of time, and then deflate the pressure to release the gripper. It is noteworthy that in power grasping process the hand pose is invariable. Mimicking the human behavior and based on previously described contact 
detection algorithm size recognition algorithm are introduced in Algorithm [2].

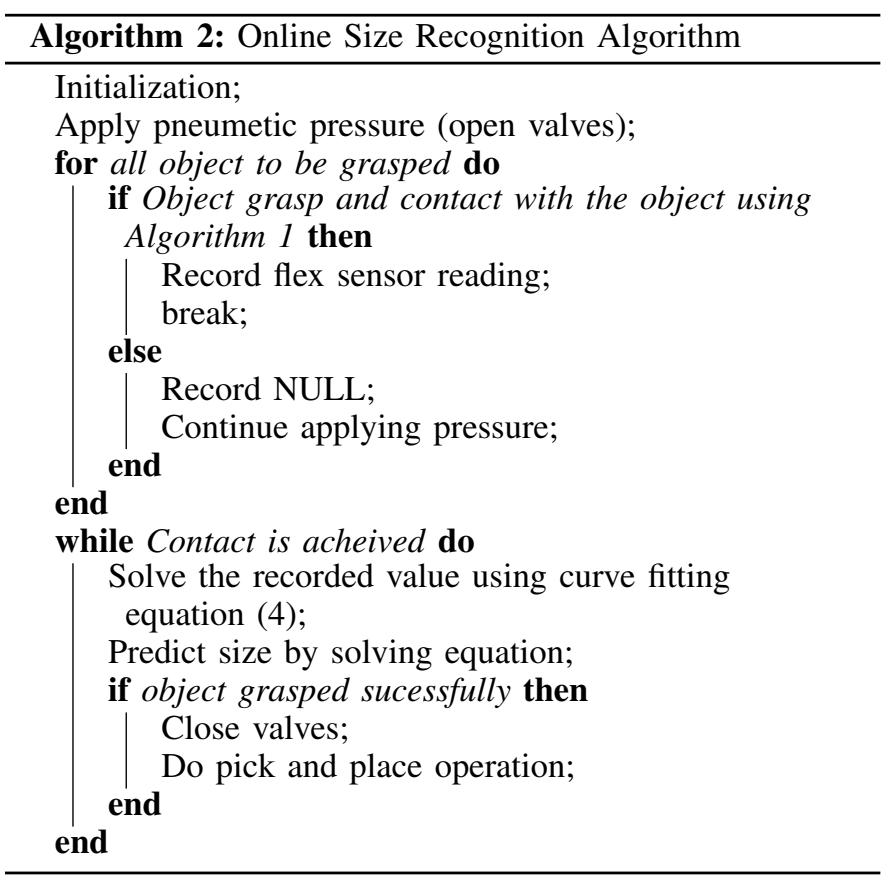

\section{EXPERIMENTS}

\section{A. Contact Detection and Validation}

In the proposed contact detection algorithm (Algorithm1), a series of contact detection experiments are performed and analyzed by considering the numerical gradient for a sample set of flex sensor readings. It has been noted that during contact occurrence with the object the the numerical gradient of bending value changes drastically. In literature, contact detection was performed by comparing with free grasping but there is always a chance of nonlinear data that may give a wrong contact detection. Moreover, the proposed contact detection algorithm is validated using a touch sensor attached to the fingertip of the gripper. An envelope grasping is performed using a flex sensor and touch sensor simultaneously. When the fingertip contacts with the object, the touch sensor gives a high digital signal as shown in Fig. 7. It is observed that the contact point achieved by the proposed contact detection algorithm and touch sensor are approximately the same.

\section{B. Size Recognition Implementation}

The exclusive behavior of flex sensor response with different size objects not only demonstrates repeatability of contact detection but also shows the capability of size recognition. A set of 3D printed PLA spheres (error $\pm 0.01 \mathrm{~mm}$ ) of diameter from $50 \mathrm{~mm}$ to $104 \mathrm{~mm}$ with an interval of $6 \mathrm{~mm}$ is considered as shown in Fig. 8. Mimicking the human grasping behavior [19], envelope grasping is performed using Algorithm 2.

By considering a constant value of source pressure (i) envelope grasping are performed (ii) contact point flex sensor value of these 9 objects is collected using the Algorithm1 (iii) curve is fitted to flex sensor readings and measured object size.

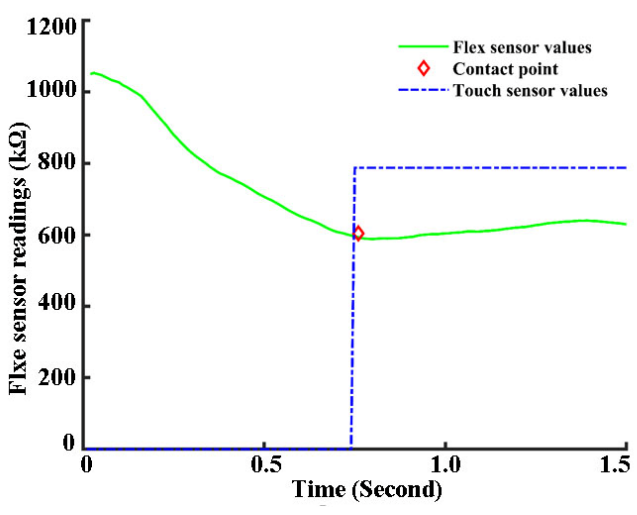

Fig. 7. Contact Detection Validation using Touch Sensor

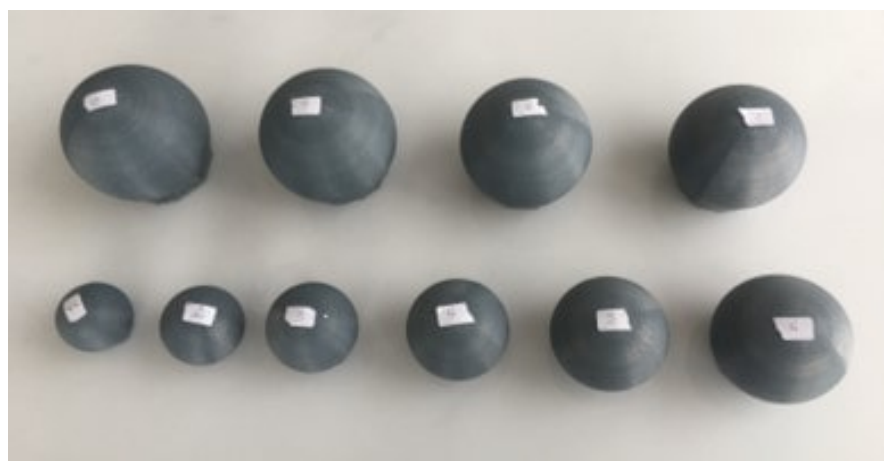

Fig. 8. Set of Different Size PLA Spheres

Fig. 9 depicts the curve fitting graph of polynomial degree 2 using solving equation (4):

$$
f(x)=0.0004 x^{2}-0.09528 x+37.98
$$

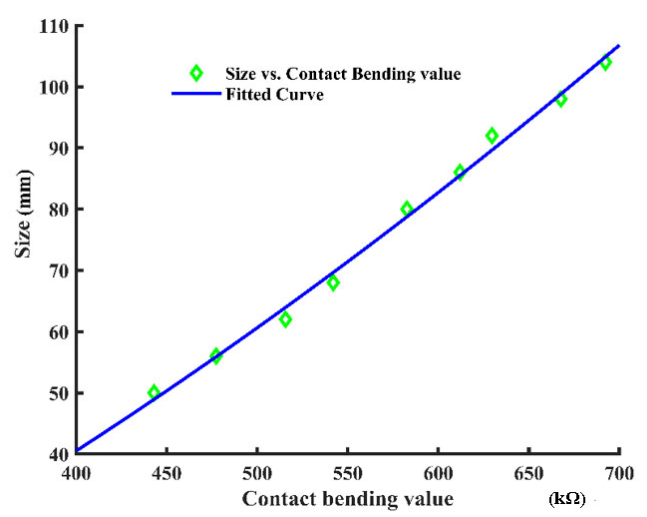

Fig. 9. Curve Fitting of Polynomial Degree 2

Where $x$ is the data from the contact flex sensor obtained with the aid of Algorithm1. The fitted polynomial curve of degree 2 obtained from the proposed method, provides better $\mathrm{R}$-square value as compared to previous work [18]. By solving the equation (4), the size of the object can be predicted. 


\section{Grasping State}

Sensitive objects handling is a crucial task in the automated industry because during grasping it can degrade the quality of the objects. Feedback of grasping state i.e. whether the object is grasped or not is a challenging task. In this section, the grasping state has been analyzed using raw data of sensory feedback. Fig. 10 shows the response of the successful and unsuccessful grasping state of an object. During successful grasping, after contact occurrence, the numerical gradient tends to increase constantly whereas during unsuccessfully grasping gradient value is decreased abruptly. This type of information can be useful to the robot as a feedback grasping confirmation of the object. Furthermore, it can be implemented in humanrobot interaction where a robot may acquire the information of the object is taken by humans or not which may save the delay in the interaction process.

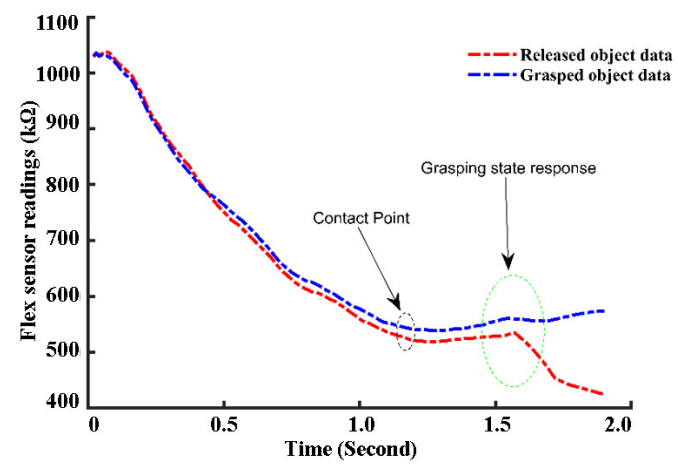

Fig. 10. Comparison of Successful and Unsuccessful Grasp State of the Target Object

\section{TESt Results}

\section{A. Size Recognisation}

A suitable source pressure $(172 \mathrm{kPa})$ is provided to perform the testing of size recognition. When the soft pneumatic gripper contacts with the objects, it starts grasping. Based on Algorithm 2 online size recognition has been performed by utilizing the curve fitting equation. A PLA sphere of $74 \mathrm{~mm}$ diameter is choosen to perform envelope grasping, which demonstrates the repeatability of the size recognition algorithm. It can be noticed from Table I, that the prediction error of the real size and predicted size is within $\pm 3 \mathrm{~mm}$. Moreover, the size recognition algorithm is tested with sensitive fruits, vegetables and daily life objects of three different sizes respectively as depicted in Fig. 11. The prediction error is presented in Table II, which shows the diverse application of the proposed size recognition algorithm regardless type of material.

From the above table it can be analysed that proposed contact detection and size recognition algorithm performs well compared with [18]. Proposed work is a less complex and easy to implement.
TABLE I. REPAETABILITY ANALYSIS

\begin{tabular}{|c|c|c|c|}
\hline \multirow{2}{*}{ ExperimentNo } & \multicolumn{3}{|c|}{ Error analysis and repeatability analysis of an object } \\
\cline { 2 - 4 } & Real Size $(\mathbf{m m})$ & Predicted Size(mm) & Errors(mm) \\
\hline 1 & 74 & 73.65 & 0.35 \\
\hline 2 & 74 & 74.54 & 0.54 \\
\hline 3 & 74 & 72.98 & 1.02 \\
\hline 4 & 74 & 73.43 & 0.57 \\
\hline 5 & 74 & 73.83 & 0.1 \\
\hline 6 & 74 & 72.54 & 1.46 \\
\hline 7 & 74 & 73.20 & 0.8 \\
\hline 8 & 74 & 72.76 & 1.24 \\
\hline
\end{tabular}

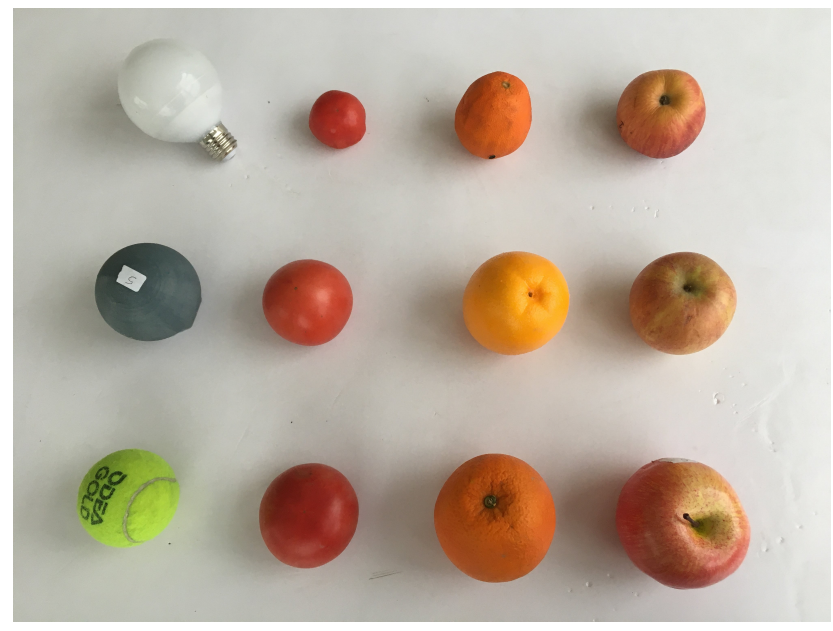

Fig. 11. Test Object for Size Recognition

\section{APPLICATION}

\section{A. Fruit Sorting Process}

After successful testing of size recognition algorithm, an automated fruit sorting environment is created (i) a sawyer robot for performing pick and place operation (ii) different size of tomatoes, oranges or apples are kept on a transport belt for handling and sorting process (iii) SONAR sensor is used to detect the object at picking position on transport belt (iv) A

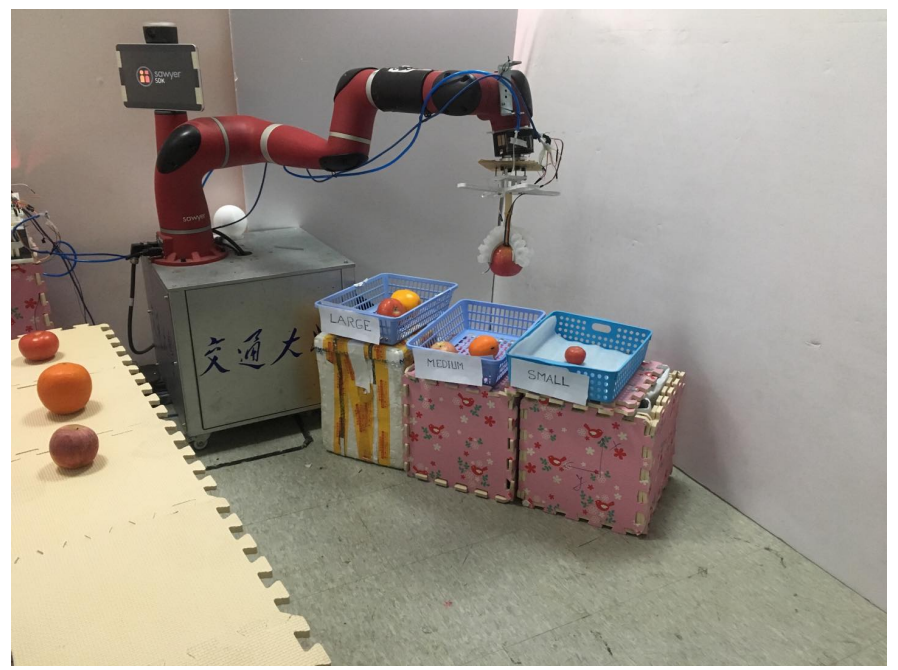

Fig. 12. Online Size Recognition and Fruit Sorting Process 
TABLE II. ONLINE SIZE RECOGNITION

\begin{tabular}{|c|l|c|c|c|}
\hline $\begin{array}{c}\text { Experiment } \\
\text { No }\end{array}$ & \multicolumn{4}{|c|}{ Size recognition and error analysis of test objects } \\
\cline { 2 - 5 } & Test object & $\begin{array}{c}\text { Real size } \\
(\mathbf{m m})\end{array}$ & $\begin{array}{c}\text { Predicted } \\
\text { size }(\mathbf{m m})\end{array}$ & $\begin{array}{c}\text { Errors } \\
(\mathbf{m m})\end{array}$ \\
\hline 1 & Tomato & 79.03 & 80.42 & 1.39 \\
\hline 2 & Tomato & 73.53 & 75.34 & 1.81 \\
\hline 3 & Tomato & 50.20 & 48.33 & 1.87 \\
\hline 4 & Orange & 86.69 & 89.12 & 2.43 \\
\hline 5 & Orange & 66.29 & 65.75 & 0.54 \\
\hline 6 & Orange & 76.48 & 78.51 & 2.03 \\
\hline 7 & Apple & 86.54 & 88.40 & 1.86 \\
\hline 8 & Apple & 70.70 & 69.23 & 1.47 \\
\hline 9 & Apple & 77.68 & 78.65 & 0.97 \\
\hline 10 & Tennis Ball & 64.08 & 65.97 & 1.89 \\
\hline 11 & Led Bulb & 78.59 & 76.58 & 2.01 \\
\hline
\end{tabular}

FSR sensor is attached at the center position of the lower base of the gripper (v) ROS with Matlab is used to implement the proposed algorithm. The fruits placed on the transport belt are continuously moving toward the robot picking position. When the SONAR sensor detects the object, the transportation belt is stopped and the manipulator starts moving towards the objects to be picked. When FSR sensor touches the object to be picked, manupulator stops and grasping operation is performed [18]. The grasping is performed by sending command from Matlab as a rosnode and also monitors the real-time feedback of the flex sensor. After the successful online implementation of the proposed contact detection and size recognition algorithm, the robot manipulator is planned to place the object into their respective bins. This process is performed for a series of objects as shown in Fig. 12.

\section{CONCLUSION}

Sensitive objects handling is a crucial task in the automated industry because during grasping it can degrade the quality of the objects such as fruits, vegetables, and daily life items. This paper demonstrates the implementation of simple sensory feedback during grasping which can be used in the handling process of objects. A numerical gradient-based algorithm is proposed and validated to detect the contact occurrence of the target object. Based on the contact detection algorithm, a size recognition algorithm is proposed which is implemented to perform an automated object sorting process. An experimental setup is developed using the sawyer robot controlled using ROS and MATLAB to implement the proposed technique. It has been noted that the proposed contact detection method has shown better accuracy $( \pm 3 \mathrm{~mm})$ in terms of size recognition as compared with previous work. Moreover, successful and unsuccessful grasping state of objects has been analysed using raw flex sensor data which can be utilized in human-robot interaction to increase efficiency in the automation process. The key benefits of the proposed algorithm are less complexity, better accuracy, and lower cost.This research can be extended using a wider set of objects with more variation and grasp orientation. Multi-segment flex sensor may provide better sensitivity and accuracy.

\section{REFERENCES}

[1] "Guest editorial focused section on soft actuators, sensors, and components (sasc)," IEEE/ASME Transactions on Mechatronics, vol. 24, no. 1, pp. 1-4, Feb 2019
[2] Z. Wang, Y. Torigoe, and S. Hirai, "A prestressed soft gripper: Design, modeling, fabrication, and tests for food handling," IEEE Robotics and Automation Letters, vol. 2, no. 4, pp. 1909-1916, Oct 2017.

[3] P. Polygerinos, N. Correll, S. A. Morin, B. Mosadegh, C. D. Onal, K. Petersen, M. Cianchetti, M. T. Tolley, and R. F. Shepherd, "Soft robotics: Review of fluid-driven intrinsically soft devices; manufacturing, sensing, control, and applications in human-robot interaction," Advanced Engineering Materials, vol. 19, no. 12, p. 1700016, 2017.

[4] J. Avery, M. Runciman, A. Darzi, and G. P. Mylonas, "Shape sensing of variable stiffness soft robots using electrical impedance tomography," in 2019 International Conference on Robotics and Automation (ICRA), May 2019, pp. 9066-9072.

[5] "A dual-mode soft gripper for food packaging," Robotics and Autonomous Systems, vol. 125, p. 103427, 2020.

[6] K. Elgeneidy, G. Neumann, S. Pearson, M. Jackson, and N. Lohse, "Contact detection and size estimation using a modular soft gripper with embedded flex sensors," in 2018 IEEE/RSJ International Conference on Intelligent Robots and Systems (IROS). IEEE, 2018, pp. 498-503.

[7] A. D. Marchese, C. D. Onal, and D. Rus, "Autonomous soft robotic fish capable of escape maneuvers using fluidic elastomer actuators," Soft Robotics, vol. 1, no. 1, pp. 75-87, 2014.

[8] Z. Zhang, A. Petit, J. Dequidt, and C. Duriez, "Calibration and external force sensing for soft robots using an rgb-d camera," IEEE Robotics and Automation Letters, vol. 4, no. 3, pp. 2356-2363, 2019.

[9] C. Choi, W. Schwarting, J. DelPreto, and D. Rus, "Learning object grasping for soft robot hands," IEEE Robotics and Automation Letters, vol. 3, no. 3, pp. 2370-2377, 2018.

[10] Q. Hao, Z. Li, H. Yan, G. Li, and B. Su, "A natural orifice soft robot with novel driven method for minimally invasive surgery (mis)," in 2017 2nd Asia-Pacific Conference on Intelligent Robot Systems (ACIRS). IEEE, 2017, pp. 289-294.

[11] B. S. Homberg, R. K. Katzschmann, M. R. Dogar, and D. Rus, "Haptic identification of objects using a modular soft robotic gripper," in 2015 IEEE/RSJ International Conference on Intelligent Robots and Systems (IROS). IEEE, 2015, pp. 1698-1705.

[12] J. A. Rogers, T. Someya, and Y. Huang, "Materials and mechanics for stretchable electronics," science, vol. 327 , no. 5973, pp. 1603-1607, 2010.

[13] S. Li, H. Zhao, and R. F. Shepherd, "Flexible and stretchable sensors for fluidic elastomer actuated soft robots," MRS Bulletin, vol. 42, no. 2, pp. 138-142, 2017.

[14] J. M. Butt, H. Wang, and R. Pathan, "Design, fabrication, and analysis of a sensorized soft robotic gripper," in 2018 IEEE 8th Annual International Conference on CYBER Technology in Automation, Control, and Intelligent Systems (CYBER), July 2018, pp. 169-174.

[15] G. Saggio, F. Riillo, L. Sbernini, and L. R. Quitadamo, "Resistive flex sensors: a survey," Smart Materials and Structures, vol. 25, no. 1, p. 013001, 2015.

[16] C. Schlagenhauf, D. Bauer, K.-H. Chang, J. P. King, D. Moro, S. Coros, and N. Pollard, "Control of tendon-driven soft foam robot hands," in 2018 IEEE-RAS 18th International Conference on Humanoid Robots (Humanoids). IEEE, 2018, pp. 1-7.

[17] K. Elgeneidy, N. Lohse, and M. Jackson, "Bending angle prediction and control of soft pneumatic actuators with embedded flex sensors-a data-driven approach," Mechatronics, vol. 50, pp. 234-247, 2018.

[18] Y. Chen, S. Guo, C. Li, H. Yang, and L. Hao, "Size recognition and adaptive grasping using an integration of actuating and sensing soft pneumatic gripper," Robotics and Autonomous Systems, vol. 104, pp. 14-24, 2018.

[19] X. Chen, Z. Li, Y. Wang, and J. Liu, "Effect of fruit and hand characteristics on thumb-index finger power-grasp stability during manual fruit sorting," Computers and electronics in agriculture, vol. 157, pp. 479-487, 2019. 